\title{
Erratum
}

\section{Magnetic field amplification in Tycho and other shell-type supernova remnants}

\author{
H. J. Völk ${ }^{1}$, E. G. Berezhko², and L. T. Ksenofontov²
}

\footnotetext{
1 Max-Planck-Institut für Kernphysik, Postfach 103980, 69029 Heidelberg, Germany e-mail: Heinrich.Voelk@mpi-hd.mpg.de

2 Yu. G. Shafer Institute of Cosmophysical Research and Aeronomy, 31 Lenin Ave., 678980 Yakutsk, Russia e-mail: [berezhko;ksenofon]@ikfia.ysn.ru
}

Key words. plasmas - acceleration of particles - ISM: supernova remnants - ISM: cosmic rays - radiation mechanisms: non-thermal errata, addenda

The full three-dimensional emissivity is assumed to have the form $q_{v}\left(\epsilon_{v}, r\right)=q_{2}\left(\epsilon_{v}\right) \exp \left[\left(r-R_{s}\right) / l_{2}\right]$. This correctly defines $q_{2}$ in Eq. (1) on page 231.

In addition, Eq. (1) has been incorrectly written. The correct equation is

$J_{v}=2 q_{2} \frac{R_{\mathrm{s}} l_{2}}{\sqrt{R_{\mathrm{s}}^{2}-\rho^{2}}}\left\{1-\frac{l_{2}}{R_{\mathrm{s}}}\left(\frac{R_{\mathrm{s}}^{2}-2 \rho^{2}}{R_{\mathrm{s}}^{2}-\rho^{2}}\right)-\exp \left(\frac{\rho^{2}-R_{\mathrm{s}}^{2}}{R_{\mathrm{s}} l_{2}}\right)\left[1-\frac{l_{2}}{R_{\mathrm{s}}}\left(\frac{R_{\mathrm{s}}^{2}-2 \rho^{2}}{R_{\mathrm{s}}^{2}-\rho^{2}}\right)+\left(\frac{R_{\mathrm{s}}^{2}-\rho^{2}}{2 R_{\mathrm{s}} l_{2}}+1\right)\left(\frac{2 \rho^{2}-R_{\mathrm{s}}^{2}}{R_{\mathrm{s}}^{2}}\right)\right]\right\}$. 